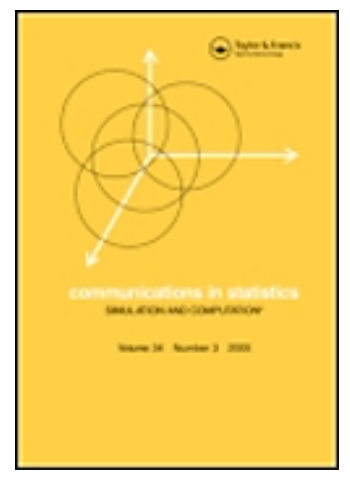

\title{
Comparison of times series with unequal length in the frequency domain
}

\begin{tabular}{|c|c|}
\hline Journal: & Communications in Statistics - Simulation and Computation \\
\hline Manuscript ID: & LSSP-2008-0213.R1 \\
\hline Manuscript Type: & Original Paper \\
\hline $\begin{array}{r}\text { Date Submitted by the } \\
\text { Author: }\end{array}$ & 07-Oct-2008 \\
\hline Complete List of Authors: & $\begin{array}{l}\text { Caiado, J.; CEMAPRE/ISEG } \\
\text { Crato, N.; CEMAPRE/ISEG } \\
\text { Peña, Daniel; Universidad Carlos III de Madrid }\end{array}$ \\
\hline Keywords: & $\begin{array}{l}\text { Cluster analysis, Time series, Spectral analysis, Interpolated } \\
\text { periodogram, Zero-padding, Reduced periodogram }\end{array}$ \\
\hline Abstract: & $\begin{array}{l}\text { In statistical data analysis, it is often important to compare, } \\
\text { classify, and cluster different time series. For these purposes } \\
\text { various methods have been proposed in the literature, but they } \\
\text { usually assume time series with the same sample size. In this } \\
\text { paper, we propose a spectral domain method for handling time } \\
\text { series of unequal length. The method makes the spectral estimates } \\
\text { comparable by producing statistics at the same frequency. The } \\
\text { procedure is compared with other possible methods by a Monte } \\
\text { Carlo simulation study. As an illustrative example, the proposed } \\
\text { method is applied to cluster industrial production series of some } \\
\text { developed countries. }\end{array}$ \\
\hline
\end{tabular}

\section{s ScholarONE" Manuscript Central}




\title{
Comparison of times series with unequal length in the frequency domain
}

\author{
Jorge Caiado ${ }^{1 *}$ Nuno Crato $^{1}$ and Daniel Peña ${ }^{2}$ \\ ${ }^{1}$ CEMAPRE/ISEG, Technical University of Lisbon, \\ Rua do Quelhas 6, 1200-781 Lisboa, Portugal. \\ ${ }^{2}$ Department of Statistics, Universidad Carlos III de Madrid, \\ Calle Madrid 126, 28903 Getafe, Spain.
}

October 7, 2008

\begin{abstract}
In statistical data analysis it is often important to compare, classify, and cluster different time series. For these purposes various methods have been proposed in the literature, but they usually assume time series with the same sample size. In this paper, we propose a spectral domain method for handling time series of unequal length. The method make the spectral estimates comparable by producing statistics at the same frequency. The procedure is compared with other methods proposed in the literature by a Monte Carlo simulation study. As an illustrative example, the proposed spectral method is applied to cluster industrial production series of some developed countries.
\end{abstract}

Keywords: Autocorrelation function; Cluster analysis; Interpolated periodogram; Reduced periodogram; Spectral analysis; Time series; Zero-padding.

\section{Introduction}

The classification and clustering of time series has useful applications in several fields. In population studies, one may be interested in identifying similarities among several series of birth and death rates. In finance, one may be interested in classifying and grouping stocks for portfolio design purposes. In international economics, one may be interested in comparing and clustering countries by looking at their main macroeconomic time series indicators.

Methods for comparing time series have been studied by using autocorrelation and spectral analysis and by model fitting methods. Building upon early work of

\footnotetext{
${ }^{*}$ E-mail contact: jcaiado@iseg.utl.pt.
} 
Coates and Diggle (1986), Diggle and Fisher (1991), Dargahi-Noubary (1992), and others, Maharaj (2002), Quinn (2006), and Caiado, Crato and Peña (2006) proposed frequency-domain methods for time series discrimination and clustering. As the last of these paper shows, spectral methods can work very well for these purposes.

A problem that often arises in real applications is dealing with time series of unequal length. For instance, in the business cycle study of some industrialized countries, Camacho, Pérez-Quiróz and Saiz (2006) deal with time series of unequal length by truncating the series to the length of the shortest one. They do it in order to use spectral estimates to compute distances across countries. In this paper, we propose to deal with this problem by adjusting the number of used periodogram ordinates. We construct an interpolated periodogram for the longer series at the frequencies defined by the shorter series. This method seems to work particularly well for comparison purposes.

The remainder of the paper is organized as follows. In Section 2, we present a well-known procedure that have been proposed in the literature for handling series of unequal length in the spectral domain; we discuss a natural extension of the usual periodogram, and propose our interpolation method. In Section 3, we present the results of a Monte Carlo simulation study where our method is compared to the other procedures. In Section 4, we apply the interpolation-periodogram based discrepancy statistic to analyze industrial time series of developed economies. In Section 5, we summarize the main results obtained in this paper.

\section{Periodogram-based discrepancy statistics}

Periodograms provide useful statistics for studying and comparing time series. Various authors have used pairwise comparison of periodogram ordinates from different series at the corresponding frequencies. This can be done directly when the series have the same number of data points. A problem arises when the data sets have different lengths and the Fourier frequencies at which the periodogram ordinates are usually computed are not the same.

Let $\left\{x_{t}, t=1, \ldots, n_{x}\right\}$ and $\left\{y_{t}, t=1, \ldots, n_{y}\right\}$ be two stationary processes with different sample sizes. Without loss of generality, assume that $n_{x}>n_{y}$. The periodogram of series $x_{t}$ is given by

$$
P_{x}\left(\omega_{j}\right)=\frac{1}{n_{x}}\left|\sum_{t=1}^{n_{x}} x_{t} e^{-i t \omega_{j}}\right|^{2}
$$

where $\omega_{j}=2 \pi j / n_{x}$, for $j=1, \ldots, m_{x}$, with $m_{x}=\left[n_{x} / 2\right]$, the largest integer less or equal to $n_{x} / 2$. The periodogram of series $y_{t}, P_{y}\left(\omega_{p}\right)$, is given by a similar expression with $\omega_{p}=2 \pi p / n_{y}$, for $p=1, \ldots, m_{y}$, with $m_{y}=\left[n_{y} / 2\right]$. When $m_{x} \neq m_{y}, \omega_{j}$ and $\omega_{p}$ do not coincide. Then, if we want to compare these two time series, a direct distance between the same periodograms ordinates cannot be computed. 
A first solution to this problem common in the pattern recognition and signal processing literature (e.g., Wang and Blostein, 2004) consists in extending the shorter series $y_{t}$, by adding zeros and getting a new series $y_{t}^{\prime}$ with the same length as the longer one. One obtains

$$
y_{t}^{\prime}= \begin{cases}y_{t}, & t=1, \ldots, n_{y} \\ 0, & t=n_{y}+1, \ldots, n_{x}\end{cases}
$$

and then computes the periodogram of series $y_{t}^{\prime}, P_{y^{\prime}}\left(\omega_{j}\right)$.This approach, called "zeropadding", matches the frequencies of both series.

A zero-padding periodogram discrepancy statistic for handling series of unequal length can then be defined by

$$
d_{Z P}(x, y)=\sqrt{\frac{1}{m_{x}} \sum_{j=1}^{m_{x}}\left[P_{x}\left(\omega_{j}\right)-P_{y^{\prime}}\left(\omega_{j}\right)\right]^{2}} .
$$

A second solution to the unequal length problem consists in calculating both periodograms at a common frequency. Although this is a simple and natural way of dealing with unequal sample sizes data, it has not been discussed in the time series classification literature. The procedure can be applied in various ways, but it seems natural to compute the periodogram of the longer series $x_{t}$ at the of the shorter series $y_{t}$ frequencies, that is

$$
P_{x}^{R P}\left(\omega_{p}\right)=\frac{1}{n_{x}}\left|\sum_{t=1}^{n_{x}} x_{t} e^{-i t \omega_{p}}\right|^{2},
$$

where $\omega_{p}=2 \pi p / n_{y}$, for $p=1, \ldots, m_{y}<m_{x}$. We will call it the "reduced periodogram".

A reduced periodogram discrepancy statistic can be defined by

$$
d_{R P}(x, y)=\sqrt{\frac{1}{m_{y}} \sum_{p=1}^{m_{y}}\left[P_{x}^{R P}\left(\omega_{p}\right)-P_{y}\left(\omega_{p}\right)\right]^{2}} .
$$

The solution we propose is to interpolate the periodogram ordinates of the series with longer length at the frequencies defined by the series with the shorter length. Without loss of generality, let $r=\left[p \frac{m_{x}}{m_{y}}\right]$ be the largest integer less or equal to $p \frac{m_{x}}{m_{y}}$ for $p=1, \ldots, m_{y}$, and $m_{y}<m_{x}$. The periodogram ordinates of $x_{t}$ can be estimated as

$$
\begin{aligned}
P_{x}^{I P}\left(\omega_{p}\right) & =P_{x}\left(\omega_{r}\right)+\left(P_{x}\left(\omega_{r+1}\right)-P_{x}\left(\omega_{r}\right)\right) \times \frac{\omega_{p, y}-\omega_{r, x}}{\omega_{r+1, x}-\omega_{r, x}} \\
& =P_{x}\left(\omega_{r}\right)\left(1-\frac{\omega_{p, y}-\omega_{r, x}}{\omega_{r+1, x}-\omega_{r, x}}\right)+P_{x}\left(\omega_{r+1}\right)\left(\frac{\omega_{p, y}-\omega_{r, x}}{\omega_{r+1, x}-\omega_{r, x}}\right) .
\end{aligned}
$$


This procedure will yield an interpolated periodogram with the same Fourier frequencies of the shorter periodogram $P_{y}\left(\omega_{p}\right)$.

The interpolated periodogram discrepancy statistic we propose is then given by

$$
d_{I P}(x, y)=\sqrt{\frac{1}{m_{y}} \sum_{p=1}^{m_{y}}\left[P_{x}^{I P}\left(\omega_{p}\right)-P_{y}\left(\omega_{p}\right)\right]^{2}} .
$$

In practical terms, if we are only interested in the dependence structure and not in the process scale, we can normalize the periodograms dividing the ordinates by the data sample variances: $N P_{x}^{I P}\left(\omega_{p}\right)=P_{x}^{I P}\left(\omega_{p}\right) / \widehat{\sigma}_{x}^{2}$ and $N P_{y}\left(\omega_{p}\right)=P_{y}\left(\omega_{p}\right) / \widehat{\sigma}_{y}^{2}$. Additionally, it is useful for the statistical analysis and testing to attain homoscedasticity in the periodogram. Since the variance of the periodogram ordinates is proportional to the spectrum at the corresponding Fourier frequencies, we may take logarithms of the ordinates. The interpolated log-normalized periodogram discrepancy statistic can then be defined as

$$
d_{I L N P}(x, y)=\sqrt{\frac{1}{m_{y}} \sum_{p=1}^{m_{y}}\left[\log N P^{I P}\left(\omega_{p}\right)-\log N P_{y}\left(\omega_{p}\right)\right]^{2}} .
$$

For reference, we also consider a well-known nonparametric discrepancy statistic based on the estimated autocorrelations (Galeano and Peña, 2000, Caiado, Crato and Peña, 2006). Let $\widehat{\rho}_{x, l}$ and $\widehat{\rho}_{y, l}$ be the sample autocorrelation functions of the longer series $x_{t}$ and shorter series $y_{t}$, respectively.

The autocorrelation discrepancy statistic is given by,

$$
d_{A C F}(x, y)=\sqrt{\sum_{l=1}^{L_{y}}\left(\widehat{\rho}_{x, l}-\widehat{\rho}_{y, l}\right)^{2}},
$$

where the number of autocorrelation lags used, $L_{y}$, would depend on the number of data points at hand. Here, $L_{y}$ will be the largest integer less or equal to $n_{y} / 10$, as recommended by Caiado, Crato and Peña (2006).

It is straightforward to show that the statistics (6), (7) and (8) fulfill some properties of a distance: (i) $d(x, y)=0$ if $P_{x}\left(\omega_{j}\right)=P_{y^{\prime}}\left(\omega_{j}\right), P_{x}^{I P}\left(\omega_{p}\right)=P_{y}\left(\omega_{p}\right)$, $N P^{I P}\left(\omega_{p}\right)=N P_{y}\left(\omega_{p}\right)$, or $\widehat{\rho}_{x, l}=\widehat{\rho}_{y, l}$; (ii) $d(x, y) \geq 0$ as all the quantities are nonnegative; and (iii) $d(x, y)=d(y, x)$, as all transformations are independent of the ordering. However, nothing guarantees the triangle inequality, which is the remaining defining property of a distance. For this reason we use the word "discrepancy" instead of "metric" as a convenient qualifier for the statistics under consideration. 


\section{Monte Carlo simulations}

To illustrate the performance of the autocorrelation and periodogram-based statistics (zero-padding, reduced and interpolated), we performed a set of Monte Carlo simulations. For each of the considered processes, we simulated pairs of series of different sample sizes, $\left(n_{1}, n_{2}\right)=\{(50,100),(200,100),(500,250),(1000,500)\}$. For each case, we performed 1000 replications. We performed the following comparisons:

(a) $\operatorname{AR}(1), \phi=0.9$ versus $\operatorname{AR}(1), \phi=0.5$;

(b) $\operatorname{AR}(1), \phi=0.9$ versus $\operatorname{ARIMA}(0,1,0)$;

(c) $\operatorname{AR}(2), \phi_{1}=0.6, \phi_{2}=-0.3$ versus $\operatorname{MA}(2), \theta_{1}=-0.6, \theta_{2}=0.3$;

(d) $\operatorname{ARFIMA}(0,0.45,0)$ versus white noise;

(e) $\operatorname{ARFIMA}(0,0.45,0)$ versus $\operatorname{AR}(1), \phi=0.95$;

(f) $\operatorname{ARFIMA}(0,0.45,0)$ versus $\operatorname{IMA}(1,1), \theta=0.4$;

(g) $\operatorname{ARMA}(1,1), \phi=0.95, \theta=0.74$ versus $\operatorname{IMA}(1,1), \theta=0.8$;

(h) Deterministic trend, $x_{t}=1+0.02 t+\varepsilon_{t}$ versus stochastic trend, $x_{t}=0.02+$ $x_{t-1}+(1-0.9 B) \varepsilon_{t}$.

In case (a), we compare low-order models of similar type and similar autocorrelation functions. In case (b), we compare a nonstationary process with a near nonstationary AR process. In case (c), we compare selected second-order ARMA processes in order to deal with peak spectra. In case (d), we compare stationary processes with very different characteristics of persistence. In case (e), we compare a near-nonstationary long-memory process with a short-memory one. In case (f), we compare a long-memory process with a nonstationary one. In case (g), we compare a near-nonstationary process with a nonstationary one. The models chosen are those discussed in Whichern (1974). In case (h), we compare a trend-stationary process and difference-stationary one. The models chosen are based on a suggestion of Enders (1995, p. 252), but incorporate a near unit root in the MA component of the stochastic formulation in order to made them more difficult to distinguish. The rational for these choices was to generate processes with similar sample characteristics. Case (d) is an apparent exception to this rule. In this case, we were simply interested in knowing whether our methods could succeed in distinguishing long memory from no memory models.

The fractional noise was simulated using the finite Fourier method of Davies and Harte (1987). The other processes were generated with the well-tested recursive method available in Matlab. In all cases, the series were generated with a zero mean and unit variance white noise. In case $(\mathrm{h})$, the series were first detrended by fitting a simple linear regression before computing the periodograms and the autocorrelation functions. As it is well known, long cyclical periods will not be eliminated by detrending.

For each case, the four generated series were grouped into two clusters by the complete linkage method. This method (also known as the farthest-neighbor method) defines the distance between two clusters by considering all possible pairs of objects 
(series), one from each cluster. The distance between two clusters is the maximum possible distance calculated for all these pairs of objects. It proceeds recursively. It starts with as many clusters as the number of series. At each step, it groups the existing clusters into fewer clusters by aggregating the two most similar ones. The procedure continues until it groups all objects. In our case, it stops with two clusters. For details, see, for instance, Johnson and Wichern (2007).

Table 1 provides the percentages of comparison successes in cases (a) to (f). Each comparison is defined as a success when the two time series of different length but generated by the same process are classified in the same group. The first rows of each cell show the results for the autocorrelations approach. The second rows of each cell show the results for the zero-padding periodogram approach. The third rows of each cell show the results for the reduced periodogram approach. The fourth rows of each cell show the results for the interpolated log normalized periodogram approach. For instance, the value 67.8 in the upper-left cell means that $67.8 \%$ of the times the two $\operatorname{AR}(1), \phi=0.9, n_{1}=50$ and $n_{2}=100$ processes were grouped into one cluster and the two $\operatorname{AR}(1), \phi=0.5, n_{1}=50$ and $n_{2}=100$ processes were grouped into another cluster using the autocorrelations method.

The interpolated-periodogram discrepancy statistic shows a remarkable good performance on the comparisons among stationary processes with ARMA and ARFIMA formulations, and shows a performance that increases significantly with the sample size on the comparison between ARMA and ARIMA processes and between ARIMA and ARFIMA processes.

The zero padding method works well for classifying longer series of similar length. However, it is not able to separate well near-nonstationary processes with large samples from nonstationary processes with short samples, and, more importantly, it does not perform well on the comparison between longer stationary and shorter nearnonstationary ARMA processes. In fact, when sample sizes are very unbalanced, the shorter series periodogram is distorted by the zero-padding method. Zero padding is equivalent to add new ordinate values that are linear combinations of the periodogram ordinates of the original series. Naturally, the resulting statistics and tests suffer from this problem.

The reduced periodogram and the ACF methods are always dominated by the other methods. In particularly, the reduced periodogram method displays a very poor performance for distinguishing similar processes with small samples and the ACF method is not able to distinguish near-nonstationary processes with large samples from nonstationary processes with short samples.

In order to better assess the methods, we have performed additional simulations for other and more dissimilar models. Results were much alike the ones here presented and pointed to the same hierarchy of discrepancy statistics. We have also explored other hierarchical and non-hierarchical clustering procedures. Results were again similar and provided the same recommendations for the discrepancy statistics choice. 

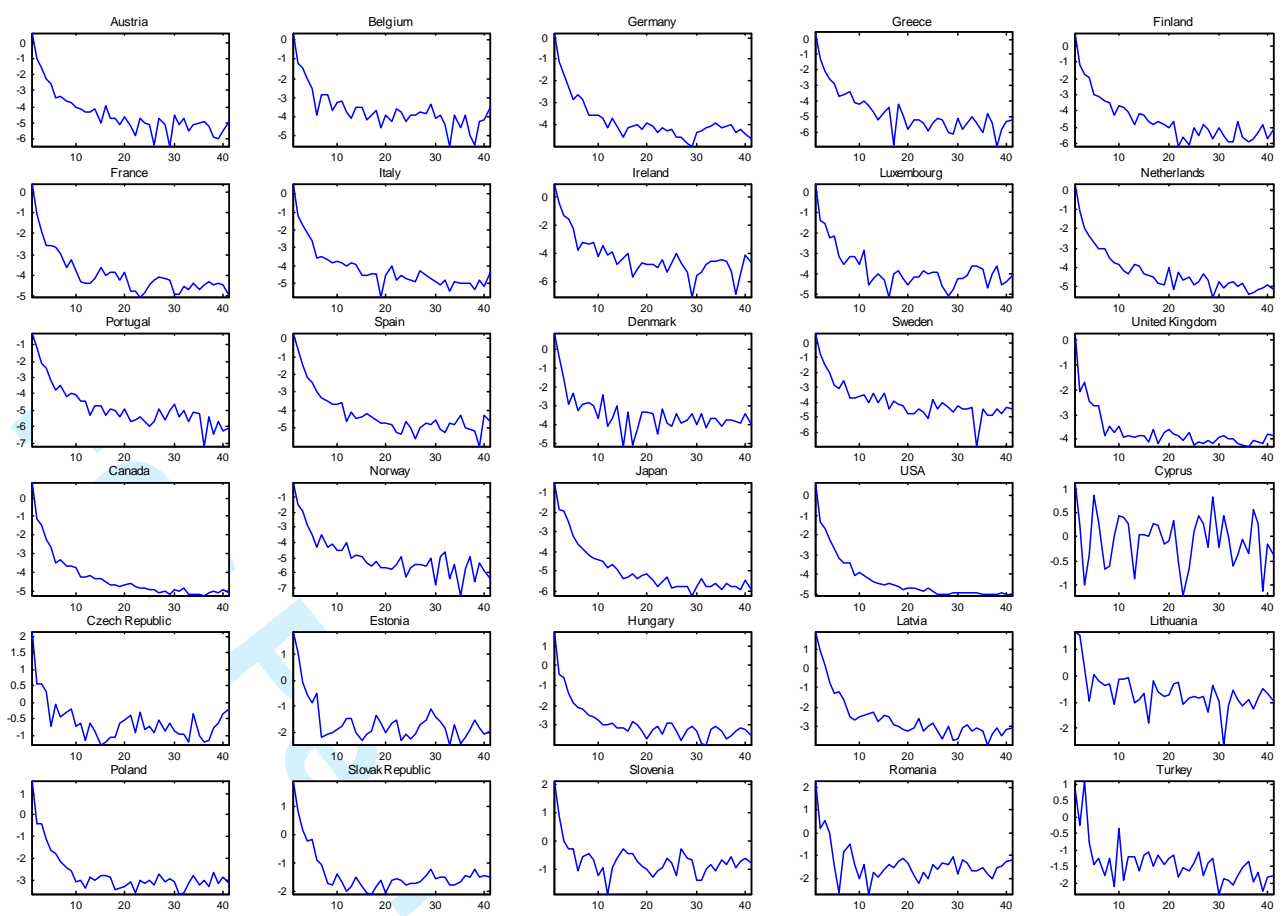

Figure 1: Log normalized interpolated periodograms of 30 European and some developed countries

\section{Application}

As an illustration of the possibilities of these techniques, we compared the industrial time series of a set of developed countries. We used monthly data of seasonally adjusted industrial production indices for a large set of European and other industrialized economies. Available data are summarized on Table 2 (source data: Camacho, Pérez-Quiróz and Saiz, 2006). For such large data set, it is unavoidable that sample periods do not coincide. In order to use all available data, it is necessary to apply techniques such as the ones we have described.

In our application, we started by computing the interpolated log normalized periodograms for each of the $k=30$ production series. The corresponding graphs are shown on Figure 1. We then computed all the corresponding $k(k-1) / 2$ pairwise distances, by using the ILNP discrepancy statistic given in (7). In order to be able to interpret resulting data, we used two well-known techniques: the multidimensional scaling and the hierarchical clustering tree, or dendrogram (see sections 12.6 and 12.3, respectively, of Johnson and Wichern, 2007, for example). 
Firstly, we used the multidimensional scaling technique, also often referred to as principal coordinates analysis, which creates a configuration of $k$ points in a lower dimensional map (usually two or three). Let $D$ be the observed $k \times k$ dissimilarity matrix, applying the multidimensional scaling to the matrix $D$ gives a $k \times s$ configuration matrix $T$, where the rows of $T$ are the coordinates values of $k$ points in $s$-dimensional representation of the observed dissimilarities for some $s<k$. The determination of the dimensionality of the spatial configuration is given by the $v$ eigenvectors of $T \times T^{\prime}$ corresponding to the largest $v$ eigenvalues.

Table 3 shows the eigenvalues resulting from distances between countries and the eigenvectors associated with the first two eigenvalues. The first eigenvalue is equal to $93.76 \%$ of the sum of all the eigenvalues. The sum of the first two eigenvalues is equal to $94.66 \%$ of the sum of all the eigenvalues. Figure 2 shows a scaling map of the derived first two coordinate values. The first dimension seems to be almost directly related to the countries' development. The second dimension is not easy to interpret. However, looking at the 2-dimensional plot and comparing the relative positions with the periodograms plots, we can make sense of some of the results. Looking at the opposite positions of Cyprus and Ireland, for instance, we realize that this distance comes from very different spectral peaks at different frequenciesthe interpolated LNP of Ireland series reaches the minimum value at frequencies $\omega_{29}=2 \pi(29) / 85=2.14367$ and $\omega_{38}=2 \pi(38) / 85=2.80895$, whereas the interpolated LNP of Cyprus series is dominated by large peaks at the same frequencies. It can also be seen that the old European Union (EU) countries (except Ireland) and the USA, Canada, Japan and Norway are close to each other and far from the new EU countries and from the then candidate countries (Estonia, Turkey, Slovak Republic, Romania, Lithuania, Slovenia, Czech Republic and Latvia). More developed Poland and Hungary are in an intermediate position.

Secondly, we consider the method of clustering the series by a hierarchical clustering tree, or dendrogram. This graphical tool shows how the clusters are combined at each stage of the procedure. We begin with each time series being considered as a separate cluster ( $k$ clusters). In the second stage, the closest two groups are linked to form $k-1$ clusters. This process continues until the last stage in which all the time series are in the same cluster.

Figure 3 shows the dendrogram for the industrial production indices series by complete linkage method, from which the clusters of countries can be identified. It can be seen at the tree that the interpolated log normalized periodogram based method can group the series into three very reasonable clusters: Cluster $1=\{\mathrm{CN}$, US, NL, IT, ES, FR, SD, BG, BD, LX, UK, DK, OE, FN, GR, IR, PT, JP, NW\}, Cluster 2 $=\{\mathrm{CY}, \mathrm{CZ}, \mathrm{SL}, \mathrm{LI}\}$ and Cluster $3=\{\mathrm{ET}, \mathrm{SK}, \mathrm{RO}, \mathrm{TK}, \mathrm{HN}, \mathrm{PO}, \mathrm{LA}\}$. Cluster 1 includes all the old EU countries and the USA, Canada, Japan and Norway. Cluster 2 grouped four new EU countries (Cyprus, Czech Republic, Slovenia and Lithuania). Cluster 3 includes the other new EU countries (Estonia, Slovak Republic, Hungary, Poland, Latvia) and the then candidate countries (Romania and Turkey). 


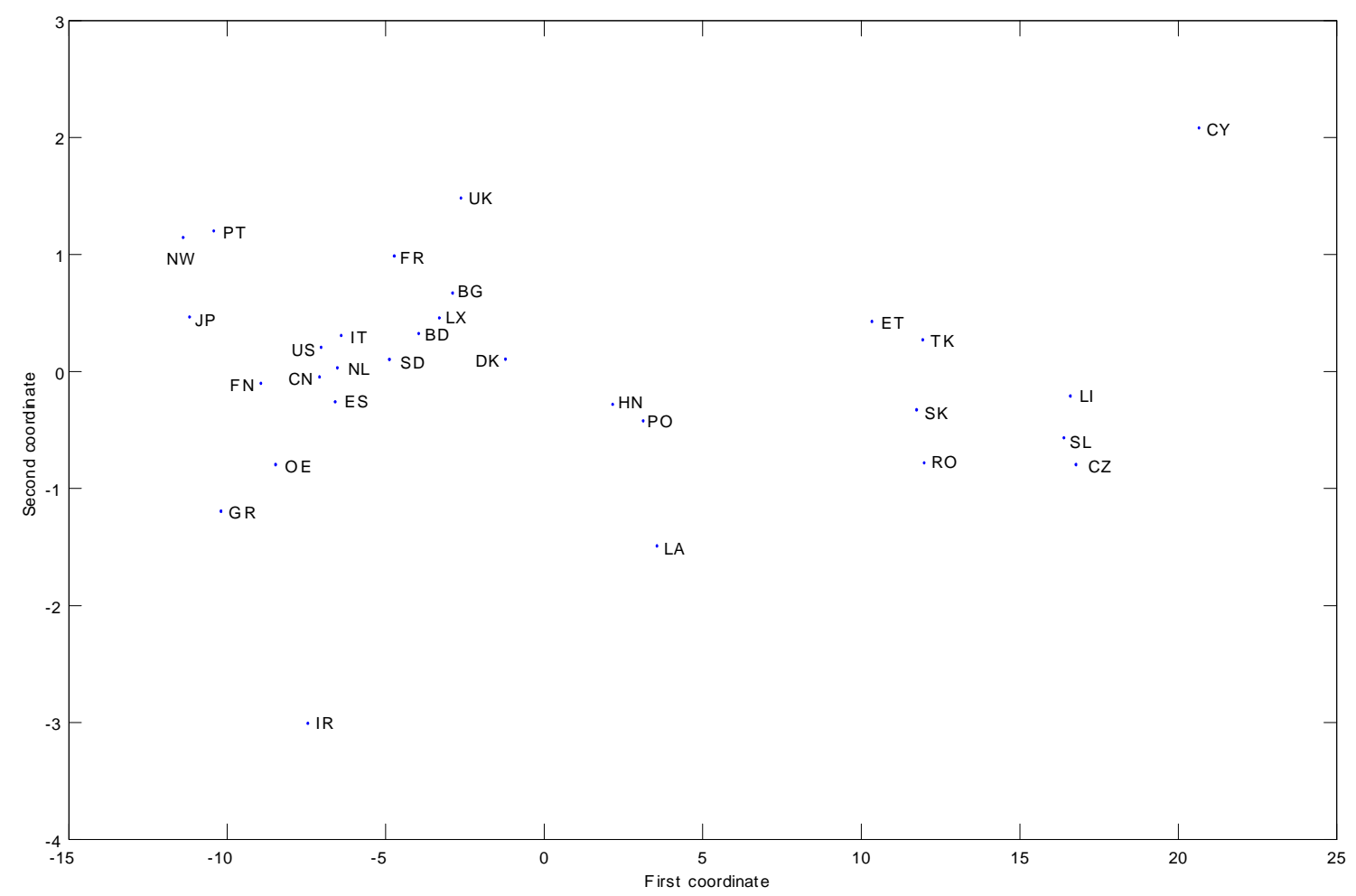

Figure 2: Multidimensional scaling for interpolated LNP distances between 30 European and some developed countries 


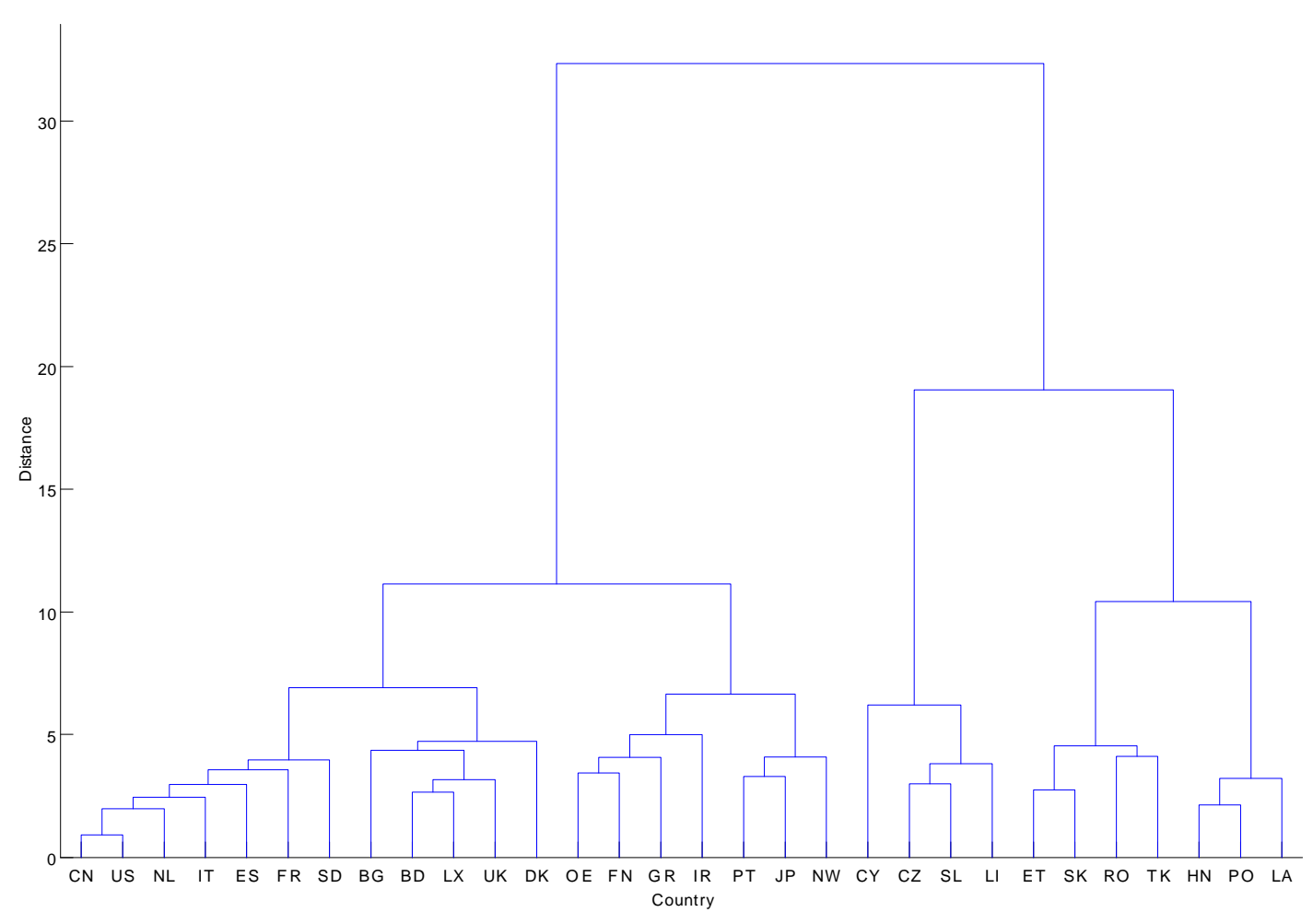

Figure 3: Complete linkage dendrogram for interpolated LNP distances between 30 European and some developed countries 
These results seem to be very reasonable. Not surprisingly, they group together the more developed countries. They essentially confirm the conclusions of Camacho, Pérez-Quiróz and Saiz (2006), adding some interesting information.

These authors also used hierarchical clustering and multidimensional scaling techniques to identify cyclical linkages among countries. From cluster analysis, they found four clusters. The first includes most of the old EU countries, the new EU countries Poland, Slovenia and Hungary, and the industrialized country Japan; the second includes the industrialized countries US, Canada, United Kingdom, and Finland; the third includes the other new EU countries (Latvia, Estonia, Czech Republic, Lithuania and Slovak Republic), the candidate countries (Romania and Turkey), and the industrialized country Norway; and the fourth includes the old European Union countries Portugal and Greece, and Cyprus. From the multidimensional scaling map, they found that most old EU member countries are close to each other and far from the new EU member countries (except Cyprus and Slovenia); and that the very industrialized countries US, Canada and United Kingdom, and new EU member countries Hungary and Finland are close to each other in a distinct location.

By using older information, our analysis is able to distinguish better the old from the new EU countries. It is also able to show US, Canada, and Japan close to the EU industrialized countries. Camacho, Pérez-Quiróz and Saiz (2006) have only used data from 1992 onwards, while we could use data from 1962 onwards. We thus confirmed some of previous results, but our method allows complementing them with more extensive data.

\section{Concluding remarks}

In this paper, we presented and discussed two spectral-discrepancy statistics for comparison, classification, and clustering analysis of time series with unequal length. We proposed a novel third statistic based on the interpolated periodogram for the same purposes. We then evaluated this latter statistic against the others. For reference, we also used an autocorrelation-based discrepancy statistic.

A simulation study indicated that the proposed method, the interpolated log normalized periodogram approach, performs very well for a wide type of comparisons: (i) different stationary processes with similar sample properties, (ii) nonstationary versus near nonstationary processes, and (iii) short-memory versus long-memory processes. Moreover, in the comparison of time series of very different sample sizes, the proposed method is preferred to the autocorrelation, the zero-padding periodogram and the reduced periodogram based methods. One application to industrial production series also demonstrates the merits of the method.

Acknowledgment: This research was supported by a grant from the Fundação para a Ciência e a Tecnologia (FEDER/POCI 2010) and by MEC project SEJ2004-03303, 
Spain. The authors gratefully acknowledge the helpful comments and suggestions of an anonymous referee on an earlier version of this paper.

\section{References}

[1] Caiado, J., Crato, N. and Peña, D. (2006). "A periodogram-based metric for time series classification", Computational Statistics \& Data Analysis, 50, 2668-2684.

[2] Camacho, M., Pérez-Quiróz, G. and Saiz, L. (2006). "Are European business cycles close enough to be just one?", Journal of Economic Dynamics and Control, 30, 1687-1706.

[3] Coates, D. S. and Diggle, P. J. (1986). "Tests for comparing two estimated spectral densities", Journal of Time Series Analysis, 7, 7-20.

[4] Dargahi-Noubary, G. R. (1992). "Discrimination between Gaussian time series based on their spectral differences", Communications in Statistics: Theory and Methods, 21, 2439-2458.

[5] Davies, R. B. and Harte, D. S. (1987). "Tests for Hurst effect", Biometrika, 74, 95-102.

[6] Diggle, P. J. and Fisher, N. I. (1991). "Nonparametric comparison of cumulative periodograms", Applied Statistics, 40, 423-434.

[7] Enders, W. (1995). Applied Econometric Time Series, Wiley, New York.

[8] Galeano, P. and Peña, D. (2000). "Multivariate analysis in vector time series", Resenhas, 4, 383-404.

[9] Johnson, R. and Wichern, D. (2007). Applied Multivariate Statistical Analysis, 6th Ed., Prentice-Hall.

[10] Maharaj, E. A. (2002). "Comparison of non-stationary time series in the frequency domain", Computational Statistics \& Data Analysis, 40, 131-141.

[11] Quinn, B. G. (2006). "Statistical methods of spectrum change detection", Digital Signal Processing, 16, 588-596.

[12] Wang, N. and Blostein, S. (2004). "Adaptive zero-padding OFDM over frequency-selective multipath channels", Journal on Applied Signal Processing, 10, 1478-1488.

[13] Whichern, D. W. (1973). "The behaviour of the sample autocorrelation function for an integrated moving average process", Biometrika, 60, 235-239. 
Table 1: Percentages of success on the comparison of pairs of simulated time series models: Autocorrelation function (ACF), Zero-padding (ZP), Reduced periodogram (RP) and Interpolated log normalized periodogram (ILNP)

\begin{tabular}{|c|c|c|c|c|c|c|c|c|c|}
\hline \multirow[b]{2}{*}{$n_{1}, n_{2}$} & \multirow[b]{2}{*}{ Discrep. } & \multicolumn{4}{|c|}{ (a) $\operatorname{AR}(1), 0.9$ vs. $\operatorname{AR}(1), 0.5$} & \multicolumn{4}{|c|}{ (b) $\operatorname{AR}(1), 0.9$ vs. $\operatorname{ARIMA}(0,1,0)$} \\
\hline & & 50,100 & 200,100 & 500,250 & 1000,500 & 50,100 & 200,100 & 500,250 & 1000,500 \\
\hline \multirow[t]{4}{*}{50,100} & $\mathrm{ACF}$ & 67.8 & 69.5 & 67.8 & 70.5 & 19.5 & 47.4 & 72.1 & 74.2 \\
\hline & $\mathrm{ZP}$ & 63.4 & 74.0 & 78.1 & 80.3 & 20.8 & 45.6 & 88.4 & 97.6 \\
\hline & $\mathrm{RP}$ & 50.1 & 52.0 & 54.2 & 51.9 & 12.5 & 31.2 & 74.2 & 92.7 \\
\hline & ILNP & 61.2 & 73.4 & 98.4 & 100.0 & 16.4 & 42.4 & 88.0 & 99.7 \\
\hline \multirow[t]{4}{*}{200,100} & $\mathrm{ACF}$ & 89.2 & 81.3 & 82.3 & 78.1 & 14.5 & 39.8 & 80.1 & 88.7 \\
\hline & $\mathrm{ZP}$ & 87.6 & 91.4 & 92.4 & 93.5 & 25.6 & 52.0 & 88.4 & 97.2 \\
\hline & $\mathrm{RP}$ & 71.3 & 76.9 & 81.4 & 82.9 & 11.0 & 31.0 & 67.6 & 89.2 \\
\hline & ILNP & 84.8 & 87.9 & 95.4 & 99.9 & 22.8 & 36.0 & 76.6 & 96.4 \\
\hline \multirow[t]{4}{*}{500,250} & $\mathrm{ACF}$ & 98.2 & 97.4 & 90.2 & 90.8 & 11.8 & 40.5 & 78.3 & 95.6 \\
\hline & $\mathrm{ZP}$ & 97.6 & 99.2 & 99.1 & 99.3 & 31.6 & 61.6 & 88.4 & 96.4 \\
\hline & $\mathrm{RP}$ & 83.6 & 93.3 & 97.2 & 98.7 & 11.9 & 25.4 & 68.7 & 88.7 \\
\hline & ILNP & 99.1 & 98.6 & 99.2 & 99.9 & 82.4 & 58.2 & 74.8 & 92.0 \\
\hline \multirow[t]{4}{*}{1000,500} & $\mathrm{ACF}$ & 98.6 & 99.7 & 98.0 & 94.6 & 12.7 & 42.8 & 78.7 & 89.3 \\
\hline & $\mathrm{ZP}$ & 98.8 & 99.6 & 100.0 & 100.0 & 36.4 & 60.0 & 84.0 & 96.8 \\
\hline & $\mathrm{RP}$ & 91.0 & 97.4 & 99.8 & 99.9 & 11.1 & 24.8 & 67.6 & 86.7 \\
\hline & ILNP & 100.0 & 100.0 & 99.9 & 100.0 & 99.8 & 96.4 & 79.4 & 89.0 \\
\hline & & (c) $\mathrm{AR}$ & $2), 0.6,-0$. & vs. $\mathrm{MA}$ & $,-0.6,0.3$ & (d) $\mathrm{A}$ & $\overline{\operatorname{FIMA}(0,}$ & $45,0)$ vs. & W.Noise \\
\hline \multirow{5}{*}{$\frac{n_{1}, n_{2}}{50,100}$} & Discrep. & 50,100 & 200,100 & 500,250 & 1000,500 & 50,100 & 200,100 & 500,250 & 1000,500 \\
\hline & $\mathrm{ACF}$ & 32.8 & 41.7 & 44.5 & 49.9 & 68.2 & 69.1 & 65.5 & 64.3 \\
\hline & $\mathrm{ZP}$ & 32.5 & 44.6 & 62.8 & 71.0 & 39.2 & 41.6 & 49.6 & 57.2 \\
\hline & $\mathrm{RP}$ & 28.7 & 31.7 & 42.4 & 41.7 & 28.5 & 31.7 & 32.8 & 34.6 \\
\hline & ILNP & 34.9 & 49.5 & 94.7 & 100.0 & 45.5 & 54.6 & 95.3 & 100.0 \\
\hline \multirow[t]{4}{*}{200,100} & $\mathrm{ACF}$ & 45.6 & 42.3 & 50.7 & 48.8 & 83.7 & 75.4 & 73.5 & 70.6 \\
\hline & $\mathrm{ZP}$ & 40.9 & 47.8 & 71.2 & 83.3 & 54.8 & 60.1 & 70.4 & 79.3 \\
\hline & $\mathrm{RP}$ & 30.8 & 39.9 & 48.9 & 54.3 & 34.8 & 41.9 & 51.3 & 49.6 \\
\hline & ILNP & 55.2 & 58.8 & 80.7 & 98.7 & 63.8 & 66.7 & 82.8 & 99.4 \\
\hline \multirow[t]{4}{*}{500,250} & $\mathrm{ACF}$ & 48.6 & 55.2 & 56.8 & 57.7 & 97.7 & 90.8 & 74.9 & 73.8 \\
\hline & $\mathrm{ZP}$ & 53.0 & 67.5 & 77.6 & 92.3 & 82.4 & 89.2 & 90.1 & 94.4 \\
\hline & $\mathrm{RP}$ & 33.2 & 47.4 & 63.6 & 73.6 & 43.7 & 57.1 & 77.0 & 83.7 \\
\hline & ILNP & 93.4 & 81.3 & 88.4 & 91.3 & 95.5 & 87.0 & 93.7 & 95.9 \\
\hline \multirow[t]{4}{*}{1000,500} & $\mathrm{ACF}$ & 50.8 & 51.6 & 65.9 & 69.7 & 99.7 & 97.8 & 87.0 & 76.3 \\
\hline & $\mathrm{ZP}$ & 57.9 & 76.1 & 91.2 & 95.7 & 90.8 & 96.4 & 98.8 & 99.1 \\
\hline & $\mathrm{RP}$ & 32.2 & 46.3 & 69.3 & 84.9 & 49.1 & 63.5 & 88.2 & 94.1 \\
\hline & ILNP & 100.0 & 98.5 & 93.3 & 98.8 & 100.0 & 99.1 & 98.2 & 99.5 \\
\hline
\end{tabular}


Table 1: (Continued)

\begin{tabular}{|c|c|c|c|c|c|c|c|c|c|}
\hline \multirow[b]{2}{*}{$n_{1}, n_{2}$} & \multirow[b]{2}{*}{ Discrep. } & \multicolumn{4}{|c|}{ (e) $\operatorname{ARFIMA}(0,0.45,0)$ vs. $\operatorname{AR}(1)$} & \multicolumn{4}{|c|}{ (f) $\operatorname{ARFIMA}(0,0.45,0)$ vs. $\operatorname{IMA}(1,1)$} \\
\hline & & 50,100 & 200,100 & 500,250 & 1000,500 & 50,100 & 200,100 & 500,250 & 1000,500 \\
\hline \multirow[t]{4}{*}{50,100} & $\mathrm{ACF}$ & 51.0 & 76.1 & 88.4 & 89.8 & 43.2 & 75.3 & 94.1 & 96.0 \\
\hline & $\mathrm{ZP}$ & 72.4 & 91.5 & 99.2 & 99.5 & 53.3 & 77.5 & 92.9 & 98.2 \\
\hline & $\mathrm{RP}$ & 53.2 & 80.7 & 94.6 & 97.8 & 30.2 & 58.7 & 83.0 & 94.7 \\
\hline & ILNP & 63.5 & 86.3 & 99.5 & 100.0 & 35.6 & 66.1 & 94.6 & 99.9 \\
\hline \multirow[t]{4}{*}{200,100} & $\mathrm{ACF}$ & 45.3 & 59.6 & 83.1 & 89.6 & 37.9 & 58.4 & 92.1 & 97.4 \\
\hline & $\mathrm{ZP}$ & 74.1 & 89.8 & 99.5 & 99.8 & 54.4 & 73.8 & 93.9 & 98.2 \\
\hline & $\mathrm{RP}$ & 54.8 & 82.4 & 97.0 & 98.9 & 25.9 & 52.9 & 81.9 & 92.9 \\
\hline & ILNP & 74.9 & 85.2 & 98.7 & 100.0 & 49.5 & 63.9 & 85.9 & 97.8 \\
\hline \multirow[t]{4}{*}{500,250} & $\mathrm{ACF}$ & 39.5 & 49.6 & 56.8 & 71.5 & 31.6 & 54.8 & 78.1 & 94.7 \\
\hline & $\mathrm{ZP}$ & 75.6 & 90.4 & 98.8 & 99.9 & 54.9 & 73.7 & 91.1 & 96.9 \\
\hline & $\mathrm{RP}$ & 53.4 & 80.7 & 97.5 & 99.6 & 25.5 & 52.7 & 83.1 & 92.4 \\
\hline & ILNP & 98.7 & 93.4 & 98.6 & 100.0 & 94.6 & 83.7 & 83.3 & 93.6 \\
\hline \multirow[t]{5}{*}{1000,500} & $\mathrm{ACF}$ & 36.8 & 44.6 & 55.6 & 53.3 & 28.8 & 50.7 & 73.7 & 82.4 \\
\hline & $\mathrm{ZP}$ & 71.3 & 90.7 & 99.1 & 100.0 & 55.3 & 68.6 & 87.3 & 96.7 \\
\hline & $\mathrm{RP}$ & 51.6 & 79.9 & 97.2 & 99.8 & 25.9 & 52.7 & 79.9 & 91.9 \\
\hline & ILNP & 100.0 & 100.0 & 99.4 & 97.8 & 100.0 & 99.5 & 92.0 & 93.7 \\
\hline & & (g) & $\overline{\mathrm{ARMA}}(1$ & 1) vs. IM & $(1,1)$ & (h) $\mathrm{D}$ & term. tr & id vs. stc & c. trend \\
\hline$n_{1}, n_{2}$ & Discrep. & 50,100 & 200,100 & 500,250 & 1000,500 & 50,100 & 200,100 & 500,250 & 1000,500 \\
\hline \multirow[t]{4}{*}{50,100} & $\mathrm{ACF}$ & 9.2 & 19.4 & 56.0 & 77.3 & 9.9 & 21.7 & 48.6 & 69.0 \\
\hline & $\mathrm{ZP}$ & 5.9 & 13.1 & 46.5 & 71.8 & 9.1 & 18.6 & 58.5 & 81.7 \\
\hline & $\mathrm{RP}$ & 12.0 & 14.4 & 28.8 & 50.9 & 10.9 & 12.8 & 15.7 & 16.9 \\
\hline & ILNP & 14.5 & 28.9 & 82.4 & 99.8 & 17.4 & 29.7 & 86.9 & 100.0 \\
\hline \multirow[t]{4}{*}{200,100} & $\mathrm{ACF}$ & 9.5 & 13.3 & 47.4 & 76.9 & 10.2 & 19.1 & 48.6 & 63.1 \\
\hline & $\mathrm{ZP}$ & 10.0 & 7.0 & 25.2 & 56.2 & 7.4 & 9.2 & 38.9 & 70.6 \\
\hline & $\mathrm{RP}$ & 11.3 & 10.2 & 31.9 & 48.2 & 12.3 & 11.3 & 18.1 & 21.3 \\
\hline & ILNP & 26.7 & 22.2 & 48.1 & 88.8 & 26.9 & 23.3 & 46.5 & 93.4 \\
\hline \multirow[t]{4}{*}{500,250} & $\mathrm{ACF}$ & 18.1 & 13.4 & 45.2 & 75.1 & 8.3 & 15.9 & 41.8 & 55.1 \\
\hline & $\mathrm{ZP}$ & 37.1 & 12.6 & 11.6 & 36.4 & 16.3 & 11.6 & 20.7 & 52.2 \\
\hline & $\mathrm{RP}$ & 12.4 & 13.8 & 26.5 & 49.0 & 13.9 & 16.2 & 20.1 & 28.3 \\
\hline & ILNP & 86.9 & 42.6 & 42.0 & 63.2 & 86.3 & 48.2 & 39.7 & 63.8 \\
\hline \multirow[t]{4}{*}{1000,500} & $\mathrm{ACF}$ & 25.3 & 14.3 & 43.8 & 70.7 & 8.2 & 14.8 & 35.7 & 46.3 \\
\hline & $\mathrm{ZP}$ & 53.1 & 21.5 & 9,5 & 30.3 & 32.7 & 18.6 & 23.1 & 41.2 \\
\hline & $\mathrm{RP}$ & 13.8 & 13.3 & 25.5 & 51.1 & 11.2 & 13.6 & 21.1 & 36.2 \\
\hline & ILNP & 100.0 & 93.1 & 54.6 & 57.4 & 100.0 & 94.8 & 56.3 & 66.5 \\
\hline
\end{tabular}


Table 2: Industrial production indices series (countries and data avaibility)

\begin{tabular}{|lccclccr|}
\hline \multicolumn{1}{|c}{ Country } & Code & Sample & $n$ & Country & Code & Sample & $n$ \\
\hline Austria & OE & $62: 01-02: 12$ & 492 & Canada & CN & $62: 01-03: 01$ & 493 \\
Belgium & BG & $62: 01-03: 01$ & 493 & Norway & NW & $62: 01-03: 01$ & 493 \\
Germany & BD & $62: 01-03: 01$ & 493 & Japan & JP & $62: 01-03: 01$ & 493 \\
Greece & GR & $62: 01-03: 01$ & 493 & USA & US & $62: 01-03: 01$ & 493 \\
Finland & FN & $62: 01-03: 01$ & 493 & Cyprus & CY & $90: 01-03: 01$ & 142 \\
France & FR & $62: 01-03: 01$ & 493 & Czech Republic & CZ & $90: 01-03: 01$ & 142 \\
Italy & IT & $62: 01-03: 01$ & 493 & Estonia & ET & $95: 01-03: 01$ & 97 \\
Ireland & IR & $75: 07-03: 01$ & 331 & Hungary & HN & $90: 01-03: 01$ & 142 \\
Luxembourg & LX & $62: 01-03: 01$ & 493 & Latvia & LA & $90: 01-03: 01$ & 142 \\
Netherlands & NL & $62: 01-03: 01$ & 493 & Lithuania & LI & $96: 01-03: 01$ & 85 \\
Portugal & PT & $62: 01-03: 01$ & 493 & Poland & PO & $90: 01-03: 01$ & 142 \\
Spain & ES & $65: 01-03: 01$ & 457 & Slovak Republic & SK & $93: 01-03: 01$ & 121 \\
Denmark & DK & $74: 01-03: 01$ & 349 & Slovenia & SL & $90: 01-03: 01$ & 142 \\
Sweden & SD & $62: 01-03: 01$ & 493 & Romania & RO & $90: 01-03: 01$ & 142 \\
United Kingdom & UK & $62: 01-03: 01$ & 493 & Turkey & TK & $90: 01-03: 01$ & 142 \\
\hline
\end{tabular}

Table 3: Eigenvalues and first two eigenvectors for interpolated LNP distances between 30 European and some developed countries

\begin{tabular}{|c|c|c|c|c|c|c|c|}
\hline \multirow{2}{*}{\multicolumn{2}{|c|}{ Eigenvalues }} & \multirow[b]{2}{*}{ Country } & \multicolumn{2}{|c|}{ Eigenvectors } & \multirow[b]{2}{*}{ Country } & \multicolumn{2}{|c|}{ Eigenvectors } \\
\hline & & & 1 & 2 & & 1 & 2 \\
\hline 2925.1 & 4.6 & Austria & -8.45 & -0.40 & Canada & -7.20 & 0.02 \\
\hline 28.3 & 3.2 & Belgium & -2.90 & 0.78 & Norway & -11.39 & 1.53 \\
\hline 21.0 & 2.3 & Germany & -4.09 & 0.21 & Japan & -11.43 & 0.44 \\
\hline 19.5 & 2.1 & Greece & -10.77 & -1.86 & USA & -7.19 & 0.20 \\
\hline 18.2 & 1.8 & Finland & -9.18 & -0.12 & Cyprus & 21.10 & 1.70 \\
\hline 13.6 & 1.7 & France & -4.87 & 0.91 & Czech Republic & 17.22 & -0.55 \\
\hline 12.7 & 1.4 & Italy & -6.70 & 0.11 & Estonia & 10.50 & 0.31 \\
\hline 11.0 & 1.2 & Ireland & -7.80 & -3.25 & Hungary & 2.34 & -0.05 \\
\hline 9.8 & 1.0 & Luxembourg & -3.48 & 0.21 & Latvia & 3.63 & -1.40 \\
\hline 9.2 & 0.9 & Netherlands & -6.58 & 0.15 & Lithuania & 16.91 & -0.45 \\
\hline 7.3 & 0.7 & Portugal & -10.51 & 1.41 & Poland & 3.28 & -0.18 \\
\hline 7.0 & 0.3 & Spain & -6.62 & -0.11 & Slovak Republic & 11.86 & -0.49 \\
\hline 6.1 & 0.2 & Denmark & -1.18 & 0.23 & Slovenia & 16.77 & -0.43 \\
\hline 5.0 & 0.1 & Sweden & -5.13 & -0.17 & Romania & 12.36 & -0.33 \\
\hline 4.6 & 0.0 & United Kingdom & -2.69 & 1.36 & Turkey & 12.19 & 0.19 \\
\hline
\end{tabular}

